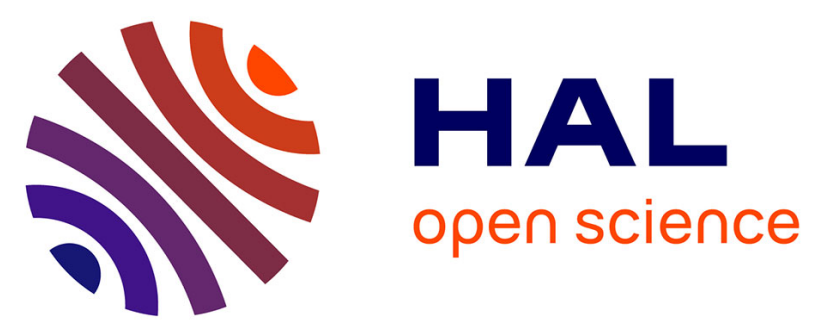

\title{
Gold Nanoparticles Assembly on Silicon and Gold Surfaces: Mechanism, Stability and Efficiency in Diclofenac Biosensing
}

Maroua Ben Haddada, Maria Hübner, Sandra Casale, Dietmar Knopp, Reinhard Niessner, Michele Salmain, Souhir Boujday

\section{To cite this version:}

Maroua Ben Haddada, Maria Hübner, Sandra Casale, Dietmar Knopp, Reinhard Niessner, et al.. Gold Nanoparticles Assembly on Silicon and Gold Surfaces: Mechanism, Stability and Efficiency in Diclofenac Biosensing. Journal of Physical Chemistry C, 2016, 120 (51), pp.29302-29311. 10.1021/acs.jpcc.6b10322 . hal-01411566

\section{HAL Id: hal-01411566 https://hal.sorbonne-universite.fr/hal-01411566}

Submitted on 7 Dec 2016

HAL is a multi-disciplinary open access archive for the deposit and dissemination of scientific research documents, whether they are published or not. The documents may come from teaching and research institutions in France or abroad, or from public or private research centers.
L'archive ouverte pluridisciplinaire HAL, est destinée au dépôt et à la diffusion de documents scientifiques de niveau recherche, publiés ou non, émanant des établissements d'enseignement et de recherche français ou étrangers, des laboratoires publics ou privés. 


\title{
Gold Nanoparticles Assembly on Silicon and Gold
}

\section{Surfaces: Mechanism, Stability and Efficiency in}

\section{Diclofenac Biosensing}

\author{
Maroua Ben Haddada ${ }^{1,3}$, Maria Huebner ${ }^{2}$, Sandra Casale ${ }^{1}$, Dietmar Knopp ${ }^{2}$, Reinhard \\ Niessner ${ }^{2}$, Michèle Salmain ${ }^{3}$, and Souhir Boujday, ${ }^{1, *}$ \\ ${ }^{1}$ Sorbonne Universités, UPMC Univ Paris 6, CNRS, Laboratoire de Réactivité de Surface \\ (LRS), F75005 Paris, France \\ ${ }^{2}$ Chair for Analytical Chemistry and Institute of Hydrochemistry, Technical University Munich, \\ Marchioninistr. 17, Munich, Germany \\ ${ }^{3}$ Sorbonne Universités, UPMC Univ Paris 06, CNRS, Institut Parisien de Chimie Moléculaire \\ (IPCM), 4 place Jussieu F-75005 Paris, France \\ Laboratoire de Réactivité de Surface, UMR CNRS 7197, Sorbonne Universités, UPMC Univ \\ Paris 6, CNRS, case 178, 4 Place Jussieu, 75252 Paris cedex 05, France \\ Tel: +33144276001, Fax: +33144276033, souhir.boujday@upmc.fr
}


ABSTRACT. We investigated the assembly of Gold nanoparticles (AuNPs) on Gold and Silicon sensors with two final objectives: (i) understanding the factors governing the interaction and (ii) building up a nanostructured piezoelectric immunosensor for diclofenac, a small-sized pharmaceutical pollutant. Different surface chemistries were devised to achieve AuNPs assembly on planar substrates. These surface chemistries included amines to immobilize AuNPs via electrostatic interaction, or a mixture of amines and thiols to covalently attach the AuNPs. We also generated PEG-amine terminated surfaces to benefit from the well-known non-biofouling properties of PEG-coated surfaces. The functional substrates and the resulting gold nanoparticle layers were characterized in detail by Surface IR, contact angle measurements and Scanning Electron Microscopy (SEM). The mechanism of adsorption is discussed herein considering the nature of the terminal groups and their charge at the $\mathrm{pH}$ of AuNPs adsorption. The coverage and the dispersion of AuNPs were strongly dependent on the anchoring points on the surfaces; the optimal were reached when the attachment layer offered multiple interaction points, in particular, for $\mathrm{NH}_{2} / \mathrm{SH}$ and $\mathrm{PEG} / \mathrm{NH}_{2}$ terminated surfaces, where the percentage of isolated particles was up to $78 \%$. In addition, PEG-coated surfaces led to a stable AuNPs layer resistant to ultrasounds and to further functionalization of the immobilized nanoparticles. These surfaces were used to engineer quartz crystal microbalance (QCM) biosensors for diclofenac detection. The AuNPs nanostructured substrates significantly enhanced the biosensor sensitivity as compared to planar substrates (up to 6 times higher). This enhancement presages a higher sensitivity in the competitive detection of diclofenac on these systems. More importantly, despite the biorecognition and the drastic regeneration conditions, SEM images show that gold nanoparticles layers are stable and reliable, which paves the way for their use as nanostructured platforms for multiple applications. 


\section{INTRODUCTION}

The assembly of gold nanoparticles (AuNPs) onto planar substrates has expanded tremendously over the last several decades, particularly in the biosensors field ${ }^{1-3}$. These nano-objects lead to dramatic improvements in the performance and sensitivity of biosensing devices owing to their plasmonic properties and to their efficiency as nanostructuration agents. The benefit from plasmonic properties of planar substrates decorated by gold nanoparticles is dramatic for techniques such as surface enhanced Raman spectroscopy (SERS) now widely employed for biosensing $^{4-6}$. The interest in these nano-objects for signal enhancement in biosensors based on non optical techniques is more recent, yet it is growing fast. In the particular case of quartz crystal microbalance (QCM) biosensors, nanostructuration of the surface of the quartz crystal electrodes with AuNPs leads to an increase of the accessible surface area and by then the number of binding sites ${ }^{7-8}$. For DNA biosensors, nanostructuration by AuNPs results in a better attachment of the oligonucleotides to the surface, up to 10 times higher than on planar substrates, and therefore an increased capacity for nucleic acid detection ${ }^{9-11}$. The same trend was observed for antibody-based biosensors; despite the bigger size of these receptors, their density was improved while preserving affinity to their target ${ }^{12-14}$. All the same, antibody fragment based biosensors benefit from AuNPs assembly; higher sensitivity and overall better immunosensing performances were evidenced ${ }^{15-16}$.

Grafting of metallic particles on substrates is often done a posteriori, starting from colloidal solutions ${ }^{4}$. Commonly synthesized by the so-called "Turkevich Synthesis ${ }^{17}$," -involving the reduction of $\mathrm{Au}$ ions by reducing agents such as citrate - gold nanoparticles are formed through a classical nucleation and growth process terminated by citrate $\left(\mathrm{Na}_{3} \mathrm{C}_{6} \mathrm{H}_{5} \mathrm{O}_{7}\right)$, which controls the nanoparticle size and morphology by acting as a surface functional layer or a capping agent, and 
preventing inter-particle aggregation through electrostatic repulsion. Prior to AuNPs deposition, surfaces need to be covered with reactive functions, usually amines or thiols, to favour the interaction with AuNPs through electrostatic or covalent bonds. Molecules commonly used at this step are organothiols in the case of gold ${ }^{18-19}$ or organosilanes for oxidized silicon and glass surfaces ${ }^{20-21}$. More recently, polymer-based coating was applied to attach AuNP on planar substrates $^{22-23}$. Poly(ethylene glycol) (PEG) is particularly adapted to biosensing applications by forming smooth and uniform PEG films that inhibit non specific adsorption ${ }^{24-25}$ and provide a high affinity to citrate-stabilized gold nanoparticles ${ }^{26-27}$. Once AuNPs are immobilized on a substrate, although the citrate capping agent is well-suited as a native functional layer during synthesis, substituting citrate, which is only bound loosely to the nanoparticle surface through weak van der Waals interactions, by a more tightly bound and well-defined capping layer is mandatory for further grafting of biomolecules or target and application in biosensing. For this post-functionalization the organosulfur compounds represent perhaps the most widely practiced route $^{28-29}$. To withstand this post-functionalization, AuNP layers should also be stable over time and their interaction with the surface strong enough to ensure that the nanoparticles remain attached during further functionalization and upon utilization. Despite the huge interest in AuNPnanostructured substrates, the assembly of gold nanoparticles on surfaces and their postfunctionalization still rely on an approximate approach since the fundamentals of the chemistry involved in the adsorption process are poorly understood ${ }^{20,30-31}$. In this contribution, we investigated the mechanism of assembly and the stability of spherical gold nanoparticles on functionalized silicon and gold substrates. To this purpose, we devised different surface chemistries to establish the key-parameters in the attachment layer that dominate the interaction with gold nanoparticles. Both gold and oxidized silicon substrates were 
modified by thiols and silanes to generate amine-terminated layers. Then, reaction of a thiolterminated carboxylic acid with the grafted amines allowed us to generate mixed thiol/amine terminated layers. In addition, we utilized diamine-PEG cross-linked to acid and epoxy groups on gold and silicon, respectively, to compare the efficiency of these films to the previous ones. The assembly of gold nanoparticles on these platforms was examined in depth by Scanning Electron Microscopy (SEM), focusing on the coverage and dispersion, and the impact of the attachment layers is discussed. We also investigated the influence of the post-functionalization agent (either an organothiol or a PEG) on the AuNP layers. Finally, the stable and well-dispersed layers are used to build piezoelectric immunosensors for the anti-inflammatory drug diclofenac and their efficiency was compared to planar surfaces. The stability of AuNP layer upon biorecognition and regeneration processes was eventually investigated by SEM.

\section{EXPERIMENTAL SECTION}

Materials: Sodium citrate, gold(III) chloride trihydrate, 3-glycidyloxypropyltrimethoxysilane (GOPTS), tannic acid, N-ethyl-N'-(3-(dimethylamino)propyl)carbodiimide hydrochloride (EDC), cysteamine. $\mathrm{HCl}$ (CEA), diclofenac sodium salt, phosphate-buffered saline (PBS) pH= 7.4, poly(ethylene glycol) bis(amine) DAPEG $_{68}(\mathrm{MW}=3000 \mathrm{Da})$, N-hydroxysuccinimide (NHS), 11-mercaptoundecanoic acid (MUA), (3-aminopropyl)triethoxysilane (APTES) (99\%), silicon wafers $<111>$ and anti-mouse $\operatorname{IgG}$ (Fc specific) antibody produced in goat were purchased from Sigma-Aldrich. Poly(ethylene glycol) bis(amine) $\mathrm{DAPEG}_{45}(\mathrm{MW}=2000 \mathrm{Da})$ was obtained from Huntsman (Rotterdam, Netherlands). Sodium dodecyl sulfate (SDS) was purchased from Fisher Scientific. The rabbit polyclonal anti-diclofenac serum was prepared in-house ${ }^{32}$, its analytical 
potential is detailed in reference ${ }^{33}$. Milli-Q water (18 M $\Omega$, Millipore, France) was used for the preparation of the solutions and for all washes. Experiments were carried out at room temperature unless otherwise stated.

Surface chemistry: Silicon wafers cut into 1 x $1 \mathrm{~cm}$ pieces were cleaned following a procedure including several washing steps and treatment by hydrochloric acid / methanol 1:1 (v/v) for $1 \mathrm{~h}$. Reactive hydroxyl groups were generated by shaking wafers in sulfuric acid for $1 \mathrm{~h}$. After washing with water, the substrates were dried under nitrogen flow ${ }^{34}$. APTES was grafted by immersing the wafers in a solution of silane in anhydrous toluene $(50 \mathrm{mM})$ and heating at $75^{\circ} \mathrm{C}$ for $24 \mathrm{~h}$. Samples were washed twice, sonicated for $10 \mathrm{~min}$ in anhydrous toluene, dried under nitrogen flow, and heated at $90^{\circ} \mathrm{C}$ for $2 \mathrm{~h}^{35}$. Surface sulfhydryl functions were generated by exposing the wafers to a solution of MUA (10 mM in EtOH previously activated with NHS (2.4 eq.) and EDC (1.2 eq.)).Grafting of GOPTS was carried out by covering wafers with GOPTS $(100 \mu \mathrm{L})$ and avoiding exposure to the atmosphere by assembling two substrates face-to-face. After $3 \mathrm{~h}$, the sandwiches were separated and sonicated first in ethanol, then in methanol and again in ethanol for $5 \mathrm{~min}$ each. They were dried under nitrogen flow and heated for $5 \mathrm{~min}$ at $85^{\circ} \mathrm{C}$. Afterwards, the wafers were treated with molten $\mathrm{DAPEG}_{45}(600 \mu \mathrm{L})$ and incubated at $98^{\circ} \mathrm{C}$ for $12 \mathrm{~h}$ in the sandwich format. The wafers were separated, sonicated in water for $15 \mathrm{~min}$ and dried under nitrogen flow ${ }^{25}$.

Gold-coated sensors were immersed in a freshly prepared solution of CEA (10 mM in water) or MUA (10 mM in EtOH) for 18-24 h. After washing with the same solvent, the CEA-treated surfaces were dipped into a solution of MUA (10 mM in EtOH previously activated with NHS (2.4 eq.) and EDC (1.2 eq.)) for $2 \mathrm{~h}$. The MUA-treated surfaces were exposed to $150 \mu \mathrm{L}$ of an 
aqueous solution of NHS (2.4 eq.) and EDC (1.2 eq.) for $2 \mathrm{~h}$ to activate the carboxyl groups. The surfaces were rinsed with the same solvent and dried under nitrogen flow. DAPEG 45 immobilization was finally performed as described above. All the functional substrates were stored in a dessicator.

Gold nanoparticles preparation and deposition: Colloidal gold particles were prepared according to the tannic acid method of Slot and Geuze ${ }^{36}$. To produce $100 \mathrm{~mL}$ of a $14 \mathrm{~nm}$ diameter colloid solution, two stock solutions were prepared: solution A: $1 \mathrm{~mL} \mathrm{1 \% (w/v)} \mathrm{HAuCl}_{4}$ and $79 \mathrm{~mL}$ water; solution B: $4 \mathrm{~mL} \mathrm{1 \%}$ sodium citrate, $0.025 \mathrm{~mL} \mathrm{1 \%}$ tannic acid and $16 \mathrm{~mL}$ water. Solutions A and B were heated to $60^{\circ} \mathrm{C}$ under stirring then mixed. When the solution turned red, the mixture was heated up to $95^{\circ} \mathrm{C}$ for a few minutes and cooled on ice. Colloidal solutions were kept in amber glassware, stored in the refrigerator at $4{ }^{\circ} \mathrm{C}$ and used within a month. Modified substrates were dipped into the prepared colloid solution, with no further dilution for $90 \mathrm{~min}$ under sonication (Elma, $90 \mathrm{~W}, 45 \mathrm{kHz}$ ), washed twice in water and dried under nitrogen. Surfaces were finally immersed in a solution of CEA or DAPEG 68 (1mM in EtOH) for $2 \mathrm{~h}$ to decorate the AuNPs with amino groups. The resulting substrates were stored in a dessicator.

Covalent grafting of Diclofenac: Diclofenac (10 mM in dry DMF) was activated by treatment with NHS (1.2 eq.) and EDC (1.2 eq.) for $150 \mathrm{~min}$ at room temperature. Then, the substrates terminated by amino groups were dipped into the solution, shaken over night at room temperature, washed for $15 \mathrm{~min}$ in water and dried under nitrogen flow. They were stored in a desiccator. 
Binding of anti-diclofenac antibody: The mixture of PBS and ethanol 9:1 was thoroughly degassed under vacuum (for $15 \mathrm{~min}$ ) and used as carrier buffer in the flow system (flow rate

$\left.25 \mu \mathrm{L} \mathrm{min}^{-1}\right)$. The sequence of injections was carried out as follows:

10-20 min flow of carrier buffer to stabilize the baseline signal; $10 \mathrm{~min}$ flow of 1/500 dilution of anti-diclofenac serum in PBS/EtOH; Washing with carrier buffer

The regeneration buffer was a solution of $2 \mathrm{w} \%$ sodium dodecyl sulfate (SDS) in water/ethanol $(9 / 1=\mathrm{v} / \mathrm{v})$ at $\mathrm{pH}=2(\mathrm{HCl})$.

Characterization techniques: Transmission Electron Microscopy (TEM) measurements were performed using a JEOL JEM 1011 microscope operating at an accelerating voltage of $100 \mathrm{kV}$. The TEM grids were prepared as follows: Typically $1.5 \mathrm{~mL}$ of the colloid solution was centrifuged at $11200 \mathrm{~g}$ for $10 \mathrm{~min}$ to precipitate the particles. The colorless supernatant was discarded. The residue was re-dispersed in a suitable volume of water. $2 \mu \mathrm{L}$ of this particle suspension was dispensed on a carbon-coated copper grid and dried at room temperature. Gold nanoparticles size distribution was determined using ImageJ. Scanning Electron Microscopy (SEM) of the gold and silicon substrates covered with AuNPs was performed using a FEG SU70 scanning electron microscope with a low voltage of $1 \mathrm{kV}$ at a distance of $1.9-2.3 \mathrm{~mm}$; the secondary electron detector "in Lens" was used. Images taken at different scales and in different regions of the samples were recorded. The particle densities were calculated by counting the particles in the SEM images (isolated and aggregates of 2, 3, 4, 5, 6, and $>6$ particles) on a representative area of $2.3-2.5 \mu \mathrm{m}^{2}$. For Contact Angle measurements static water contact angles were measured at room temperature using the sessile drop method and image analysis of the drop profile. The instrument, which uses a CCD camera and an image analysis processor, was 
purchased from Krüss Optronic GmbH (Hamburg, Germany). The water droplet volume was 1 $\mu \mathrm{L}$, and the contact angle $(\Theta)$ was measured $5 \mathrm{~s}$ after the drop was deposited on the sample. For each sample, the reported value is the average of the results obtained on 3 droplets and the overall accuracy in the measurements was better than $\pm 5^{\circ} . \boldsymbol{P M}-\boldsymbol{I R} \boldsymbol{R} \boldsymbol{A S}$ spectra were recorded on a commercial NICOLET Nexus FT-IR spectrometer. Details on the experimental set-up are available in reference ${ }^{37}$. Sensor chips were made of borosilicate glass substrates $(11 \mathrm{~mm} \times 11$ $\mathrm{mm}$ ), coated successively with a $2.5 \pm 1.5 \mathrm{~nm}$ thick layer of chromium and a $250 \pm 50 \mathrm{~nm}$ thick layer of gold (Arrandee, Werther, Germany). They were annealed in a butane flame to ensure a good crystallinity of the topmost layers and dipped in a bath of absolute ethanol during 15 min before adsorption. The Si wafers were analyzed by $\boldsymbol{G} \boldsymbol{A}-\boldsymbol{A T R}-\boldsymbol{I R}$ immediately after their preparation. GA-ATR-IR spectra (256 scans, $4 \mathrm{~cm}^{-1}$ resolution) were recorded on a Tensor 27 spectrometer (Bruker) equipped with a VariGATR accessory (Harrick Scientific, Pleasantville, NY) and incidence angle set at $60^{\circ}$. Quartz Crystal Microbalance with dissipation measurements $(\boldsymbol{Q C M}-\mathbf{D})$ were carried out with a 4-channel dissipative quartz crystal microbalance (Q-Sense E4) using AT-cut $5 \mathrm{MHz}$ quartz crystals (QCM sensors, $14 \mathrm{~mm}$ diameter) coated with a 3-100 $\mathrm{nm}$ layer of gold or a $50 \mathrm{~nm} \mathrm{SiO} 2$ film (Lot-oriel) at $22^{\circ} \mathrm{C}$. Prior to use, they were cleaned by ethanol and dried under nitrogen flow. Experiments were carried out in flow-through conditions using a peristaltic pump. Data were simultaneously acquired at the fundamental frequency $F$ of $5 \mathrm{MHz}$ $(N=1)$ and several overtone frequencies $(15,25,35,45$, and $55 \mathrm{MHz}$, i.e. $N=3,5,7,9$ and 11). Two physical parameters are discussed: the resonance frequency and the dissipation. The frequency change can be correlated to the mass of the adsorbed layer using the Sauerbrey equation ${ }^{38}: \Delta F=-N \Delta \mathrm{m} / \mathrm{C}_{\mathrm{f}}$, where $\mathrm{C}_{\mathrm{f}}\left(=17.7 \mathrm{ng} \mathrm{cm}^{-2} \cdot \mathrm{Hz}^{-1}\right.$ at $\left.\mathrm{F}=5 \mathrm{MHz}\right)$ is the masssensitivity constant, and $\mathrm{N}$ is the overtone number. 


\section{RESULTS AND DISCUSSION}

Synthesis and characterization of AuNPs: Citrate-stabilized spherical AuNPs were selected for this work as their synthesis has reached a level of maturity allowing a precise control of their shape, size, and dispersion. The size and dispersion of the gold colloids prepared in this work were estimated from UV-visible spectroscopy and Transmission Electron Microscopy (TEM) analyses. UV-Vis spectra and TEM images are given in the supplementary material section. On the UV-Vis spectrum (Figure S1-a), a narrow resonance plasmon band was present at $520 \mathrm{~nm}$, typical of a particle size around $15 \mathrm{~nm}^{39-40}$. This was confirmed by TEM images (Figure S1-b), mathematical treatment of the images showed a homogeneous particle size distribution with an average size of $13.5 \pm 1 \mathrm{~nm}$. The AuNP concentration in the solution, estimated by two methods (see supplementary section) was $3.6 \pm 0.2 \mathrm{nmol} \mathrm{L}^{-1}$.

Surface functionalization of Au and Si substrates: Six different surface chemistries were devised to generate a layer of AuNPs on gold and silicon planar substrates as schematized in Figure 1. Adhesion layers either included amines to ensure a strong electrostatic interaction with citrate-stabilized AuNPs ${ }^{41}$ or amines and thiols to covalently bind AuNPs through S-Au bonds. In addition, adhesion layers including $\mathrm{NH}_{2}$-terminated poly(ethylene) glycol) were built up to benefit from the well-known anti-biofouling properties of PEG-coated surfaces and prevent non specific adsorption during the immunosensing process ${ }^{27}$. We used a diamine-PEG (DAPEG 45 ) with a molecular weight of 2,000 Da, since this medium size PEG has been previously shown to provide a homogeneous cover on Si wafers ${ }^{24,26,42}$. 
Amine-terminated surfaces were generated on gold and silicon using CEA and APTES, respectively. Mixed amine/thiol terminated layers were obtained by reacting MUA on the previous amine-terminated layers. MUA reacts with approx. $24 \%$ of amine groups leading to layers with $\mathrm{SH} / \mathrm{NH}_{2}$ ratio $\sim 1 / 4{ }^{20}$. Amine-terminated PEG layers were built up by conjugating DAPEG to carboxy-decorated gold and epoxy-decorated silicon substrates that had been obtained by self-assembling of MUA and GOPTS, respectively (see Figure 1). The terminal carboxylic acid groups of MUA SAM on gold were converted into NHS esters by reaction with EDC and NHS prior to DAPEG grafting according to a previously published procedure ${ }^{25}$. The resulting surfaces are named according to the following nomenclature: Substrate (Au or $\mathrm{Si})$ - 
Terminal group $\left(\mathrm{NH}_{2}, \mathrm{NH}_{2} / \mathrm{SH}\right.$, or $\left.\mathrm{PEG}\right)$ as depicted in Figure 1.

Prior to AuNPs immobilization, the functionalized substrates were characterized by contact angle measurements and surface IR. Table 1 summarizes the static water contact angle values measured for these substrates.

Table 1 Static water contact angle measurements

\begin{tabular}{|l|l|l|l|l|}
\cline { 2 - 5 } \multicolumn{1}{c|}{} & \multicolumn{2}{l|}{ Si } & Au \\
\hline Clean & $<10^{\circ}$ & $46 \pm 2^{\circ}$ & MUA \\
\hline \multirow{2}{*}{$1^{\text {st }}$ layer } & APTES & GOPTS & CEA & $59 \pm 2^{\circ}$ \\
\cline { 2 - 5 } & $83 \pm 2^{\circ}$ & $51 \pm 1^{\circ}$ & $51 \pm 2^{\circ}$ & PEG \\
\hline \multirow{2}{*}{$2^{\text {nd }}$ layer } & APTES+MUA & PEG & CEA+MUA & $40 \pm 4^{\circ}$ \\
\cline { 2 - 5 } & $40 \pm 4^{\circ}$ & $43 \pm 2^{\circ}$ & $48 \pm 1^{\circ}$ & \\
\hline
\end{tabular}

The initial clean (oxidized) silicon surface was very hydrophilic as expected by the strong oxidizing conditions applied to the samples before analysis to generate a high density of surface hydroxyl groups ${ }^{25}$. Silanization with APTES resulted in a very large increase of the contact angle from $15^{\circ}$ to $83^{\circ}$ due to the presence of more or less ordered aminopropyl chains ${ }^{35,43}$. Further covalent binding of MUA resulted in a drop of the contact angle to $40^{\circ}$. This increase of hydrophilic character may result from a better packing of the organic layer owing to Van der Waals forces between the longer hydrocarbon chains of MUA ${ }^{44}$. Silanization with GOPTS also led to an increase of contact angle from 15 to $51^{\circ}$ as a result of organic group presence on the surface. Treatment with DAPEG slightly decreased the contact angle to $43^{\circ}$ in agreement with the known hydrophilic character of PEG derivatives ${ }^{26}$. The same trend was observed for gold surfaces, i.e. an increase of the contact angle upon CEA or MUA grafting ${ }^{45}$ and a decrease after DAPEG grafting consistent with the higher hydrophilic character expected for PEG-covered surfaces. The contact angle measured for Au-PEG was roughly the same as the one measured for Si-PEG, $\sim 40^{\circ}$.

Surface IR, either in ATR mode for silicon substrates, or PM-IRRAS mode for gold substrates, 
was used to characterize the molecular composition of the thin films deposited on both surfaces. The recorded spectra are shown in the supplementary information section (Figures S2, 3, and 4) together with the detailed band attributions (table S2). Briefly, for both substrates the characteristic bands expected upon surface modification were observed. On Au-NH $\mathrm{N}_{2}$ and $\mathrm{Si}-\mathrm{NH}_{2}$ the primary amine bands were present in the range $1660-1640 \mathrm{~cm}^{-1}$ the ammonium group bands around 1480 and $1571 \mathrm{~cm}^{-1}$. Upon MUA grafting the intensity of these bands was lower and amide bands appeared giving evidence of the successful conjugation of activated acid to surface amine groups. All the same, successful grafting of DAPEG on Au-MUA and Si-GOPTS substrates was evidenced on both systems by the presence in the IR spectra of an intense band at $1110 \mathrm{~cm}^{-1}$, assigned to the intense C-O-C stretching mode of the PEG chains ${ }^{25,46}$.

Gold nanoparticles immobilization on functionalized surfaces: The AuNPs layer attachment to the surface must be strong enough to prevent desorption/release during the successive steps of biosensor elaboration, test and regeneration. Therefore, ultrasounds were applied during AuNP deposition, to remove loosely bound nanoparticles. In addition, we have previously observed that applying ultrasounds during deposition of AuNPs on Si surfaces prevented the formation of aggregates 20 .

Scanning electron microscopy was used to estimate the coverage and dispersion of AuNP on the functionalized surfaces. SEM images and graphical estimation of AuNPs density and dispersion are shown in Figure 2. The fractional coverage was calculated by dividing the area covered by the $2 \mathrm{D}$ projection of the spherical AuNPs on the surface by the total area of the surface ${ }^{47}$, results are shown in table 2.

The coverage and dispersion of the nanoparticles layers were highly dependent on the structure 
of the organic adhesion film. On Au-NH $\mathrm{N}_{2}$ surfaces (Figure 2-d), AuNPs were barely detectable. Their absence is ascribable to the application of ultrasounds. Indeed, when the deposition was repeated under gentle stirring, SEM images (Supplementary information section, Figure S5) showed a small quantity (29 particles $/ \mu \mathrm{m}^{2}$ ) of gold nanoparticles on the surface, which supports the hypothesis of weak interactions between AuNPs and Au-NH $\mathrm{N}_{2}$ surface. Conversely, a relatively high density of gold nanoparticles $\left(510 \pm 15 \mathrm{x} \mathrm{NP} / \mu \mathrm{m}^{2}\right)$ was observed on $\mathrm{Si}-\mathrm{NH}_{2}$ substrates (Figure 2-a). This difference observed between Si and Au can be understood by considering the difference in amine coverage on the two substrates: while CEA forms a monolayer of more or less ordered thiols on gold ${ }^{48}$, APTES condensation on silica-like substrates leads to a low amine coverage ${ }^{35}$. Therefore on $\mathrm{Au}-\mathrm{NH}_{2}$ substrates only amine groups are present whereas on $\mathrm{Si}-\mathrm{NH}_{2}$ both silanols $(\mathrm{Si}-\mathrm{OH})$ and $\mathrm{NH}_{2}$ groups are present. Moreover, at the acidic $\mathrm{pH}$ of AuNPs solutions $(\mathrm{pH}=5.4)$, amino groups on $\mathrm{Au}-\mathrm{NH}_{2}$ are mostly positively charged, $\left(\mathrm{pK}_{\mathrm{a}}(\mathrm{CEA})=8.3^{49}\right)$, therefore, their interaction with AuNPs can be envisioned mainly through electrostatic bonds with negatively charged citrate ligands. Whereas on APTES modified surfaces, $\mathrm{pK}_{\mathrm{a}}=7.6^{50}$, and though ammonium species dominate, they coexist with silanol groups that are negatively charged at this $\mathrm{pH}$ ( silica $\mathrm{PZC}=2^{51}$ ). Silanolates may be involved in AuNP interaction with the surface as they can replace citrate ligands and directly interact with gold. Therefore, these cooperative interactions on $\mathrm{Si}-\mathrm{NH}_{2}$ would explain the huge difference observed with $\mathrm{Au}-\mathrm{NH}_{2}$. Note that despite this cooperative interaction, the coverage observed on $\mathrm{Si}-\mathrm{NH}_{2}$ layers was the lowest of all the silicon substrates under study (Figure 2 and Table 2). 


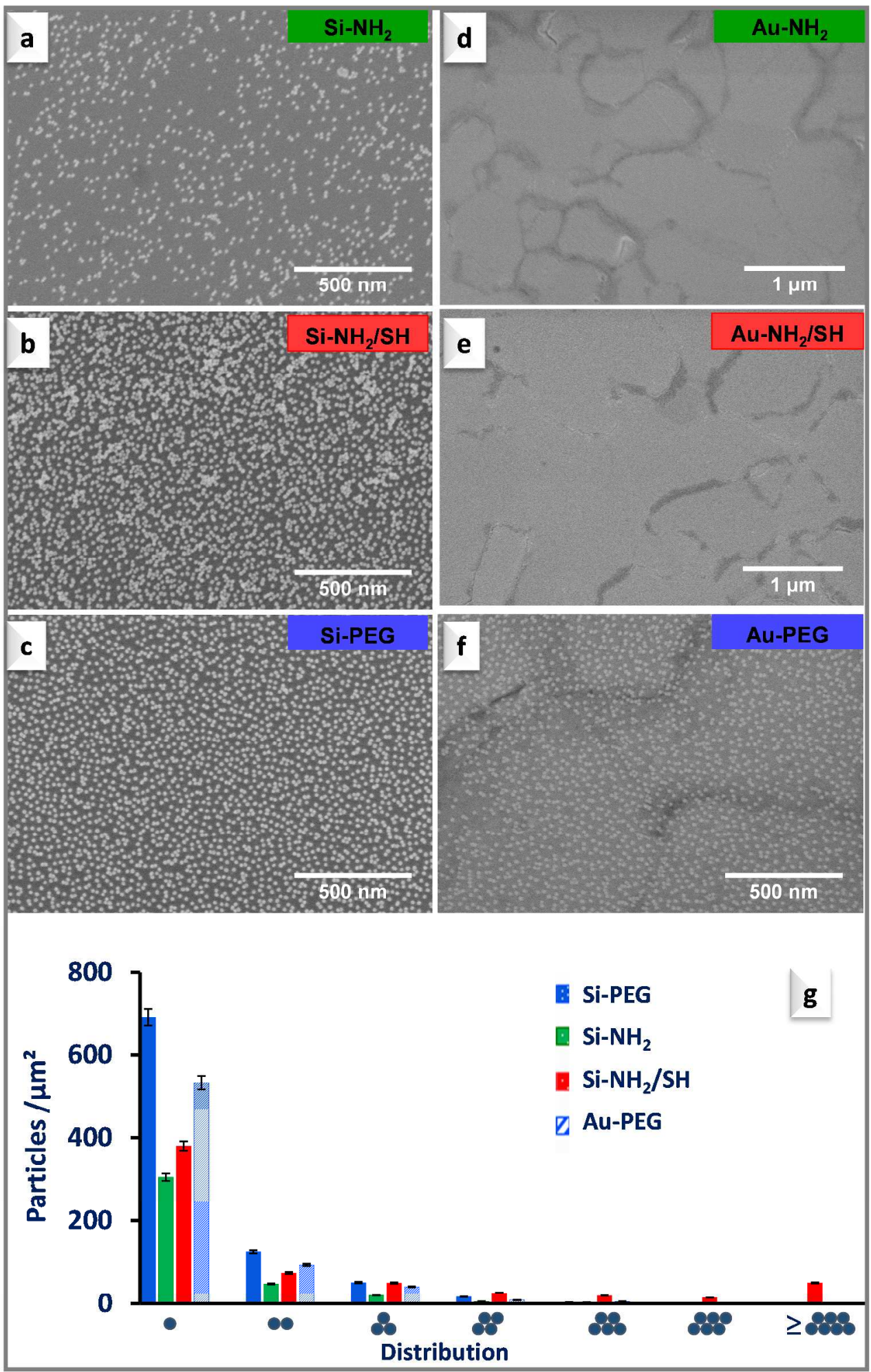

Figure 2 SEM images taken after AuNPs immobilization on the functionalized surfaces (a-f) and the resulting surface coverage $(\mathrm{g})$ 
After conjugation of MUA to the amine-terminated surfaces, the $\mathrm{Si}-\mathrm{NH}_{2} / \mathrm{SH}$ substrates exhibited a higher coverage of AuNPs (Figure 2-b). For Au- $\mathrm{NH}_{2} / \mathrm{SH}$ the improvement could not be seen on Figure 2-e, but again when the deposition was done without applying ultrasounds (Figure S5, Supplementary section) AuNPs coverage was clearly improved compared to on $\mathrm{Au}-\mathrm{NH}_{2}$. This result corroborates the efficiency of cooperative interactions towards AuNPs immobilization, here through Sulfur/Gold and ammonium/citrate interactions. On $\mathrm{Si}-\mathrm{NH}_{2} / \mathrm{SH}$, the fractional coverage in AuNPs reached 0.20 (see Table 2). However, the increase in AuNPs coverage on Si$\mathrm{NH}_{2} / \mathrm{SH}$ substrates slightly worsen their dispersion, the percentage of isolated particles was lowered to $62 \%$ and few aggregates were observed (Figure 2-b and g). The best dispersion of AuNPs and the highest surface coverage were observed for the PEGfunctionalized substrates, Si-PEG and Au-PEG (Figure 2- c and f). Both systems showed 78\% of isolated particles and fractional coverage of $0.14-0.18$ (Table 2). This difference with the previous substrates may result from the swelling properties of PEG films that make them capable of capturing citrate-stabilized AuNPs ${ }^{27}$ and trapping them by displacement of loosely bound citrate ligands and creation of multidentate interactions between the PEG chains and the Au atoms of the AuNPs ${ }^{26}$. This effect cooperates with the terminal amino group of the PEG chains that acts by electrostatic interaction with citrate ligands to stabilize the nanoparticle layers. Surprisingly, despite the thick layer of PEG $\left(1.7 \mathrm{~nm}^{25}\right)$, the substrates and/or the organic underlayers influence the coverage, as the value is $24 \%$ lower on Au-PEG than on Si-PEG. Nevertheless, though all the coverage reported in Table 2 may look low, one must keep in mind that they in the same range than the data reported in the literature where the deposition and washing conditions were less drastic, $(0.15-0.25)^{19,41,47}$. This limit is determined by the citrate ligands co-adsorbed with AuNPs that maintain an electrostatic repulsion between adsorbed 
citrate-coated AuNPs and prevent the formation of close-packed monolayer ${ }^{41,52-53}$.

Table 2. AuNPs dispersion and coverage calculated from SEM images

\begin{tabular}{llll} 
Sample & Isolated AuNPs / \% & Number of particles / $\mu \mathrm{m} 2$ & Fractional coverage \\
\hline $\mathrm{Si}-\mathrm{NH}_{2}$ & 77 & $510 \pm 20$ & 0.08 \\
$\mathrm{Si}-\mathrm{NH}_{2} / \mathrm{SH}$ & 62 & $1324 \pm 40$ & 0.20 \\
$\mathrm{Si}-\mathrm{PEG}$ & 78 & $1186 \pm 40$ & 0.18 \\
$\mathrm{Au}-\mathrm{PEG}$ & 78 & $905 \pm 30$ & 0.14
\end{tabular}

To summarize this part, offering multiple anchoring points and cooperative interactions improved the coverage and dispersion of gold nanoparticles at the surface of gold and silicon planar substrates. These systems also show a resistance to leaching upon sonication which should make them suitable for further functionalization. The PEG layers provided an excellent dispersion on both substrates (78\%). In what follows, we investigate for Si-PEG and Au-PEG substrates the stability of the AuNP layers upon post-functionalization, diclofenac biosensing and under the drastic regeneration conditions.

Post-functionalization of gold nanoparticle layers: The use of the AuNPs-modified platforms for biosensing requires the exchange of the surrounding citrate ligands by reactive functions that will be used to further construct the biosensing layer. Mastering this step is important because the AuNPs layer is mobile and ligand exchange may lead to particle aggregation or leaching from the support ${ }^{54-55}$. Two reagents were utilized for post-functionalization: CEA and PEG $_{68}$, both introduce primary amine groups for further anchoring of diclofenac via its carboxylic acid function (see molecular structure in Figure 5). These post-functionalization treatments were applied to AuNP arrays deposited on PEG-coated silicon and gold substrates. The density and dispersion of AuNPs was then estimated by SEM. Representative images and their mathematical treatment are shown in Figure 3. As expected, post-functionalization modified the AuNP dispersion and coverage. On both substrates, the overall coverage was slightly lowered. 


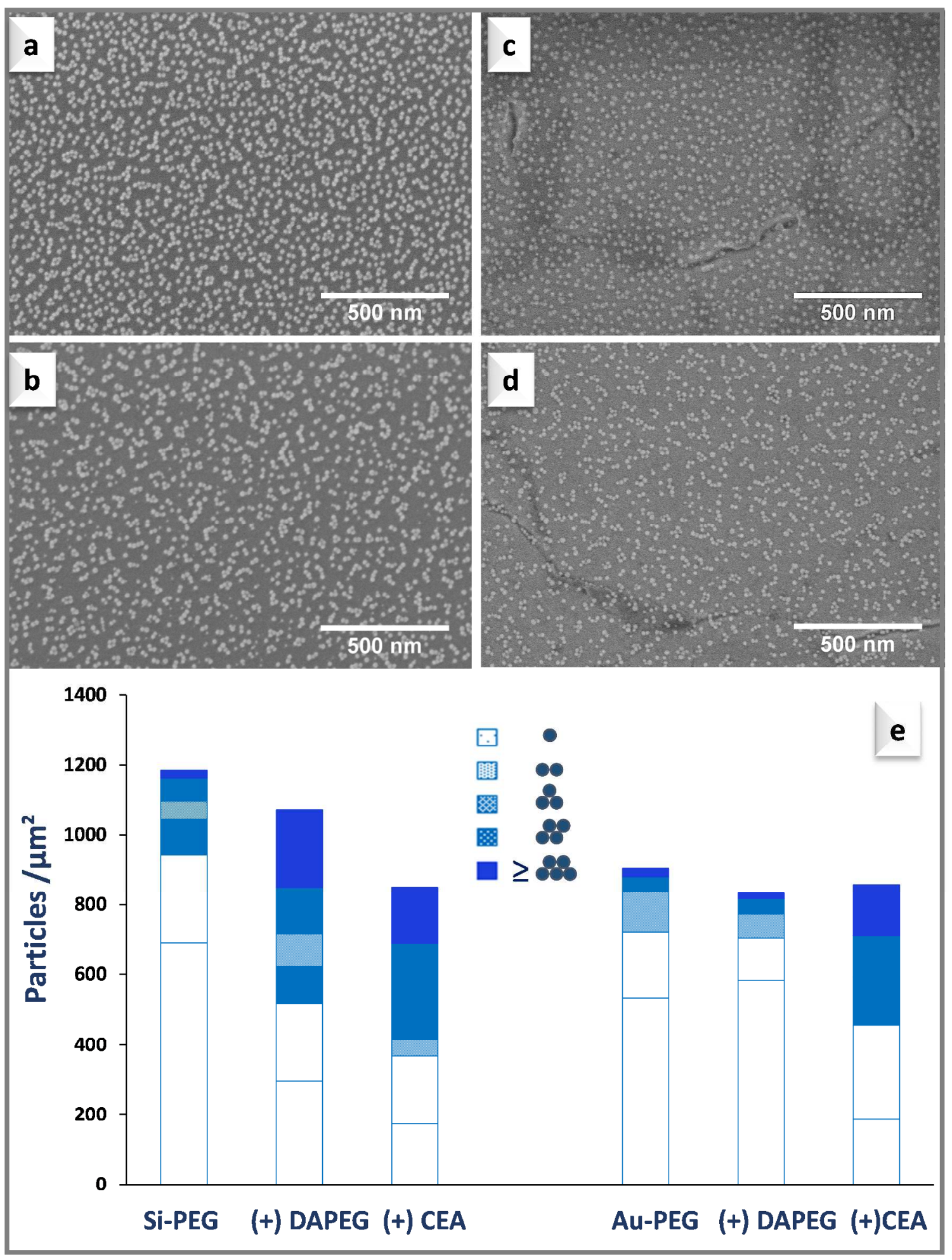

Figure 3 SEM images (a-d) and AuNP densities (e) for Si-PEG-AuNP post-functionalized by CEA (a) or PEG (b) and Au-PEG-AuNP post-functionalized by CEA (c) or PEG (d)

Post-treatment of Si-PEG-AuNP substrates by CEA or DAPEG induced both a significant 
desorption of particles (up to $30 \%$ with CEA) as well as aggregation. This behavior may be explained by the fact that, since the fractional coverage of AuNP on Si-PEG was higher than 0.15 established as the upper limit determined by Grabar et al. ${ }^{41}$ for 15 -nm diameter citrate-capped AuNPs, the exceeding AuNPs were probably more loosely bound and/or mobile so that further treatment with a weak ligand like DAPEG favored aggregation whereas further treatment with a stronger adsorbate like CEA led to both aggregation and desorption. Similar desorption was previously reported on APTES-coated silicon upon treatment with CEA and explained by the positive charge of nanoparticles upon citrate ions replacement by CEA molecules that led to repulsion from the positively charged APTES-coated surface ${ }^{52,55}$. Conversely, postfunctionalization of Au-PEG-AuNP by CEA or DAPEG did only marginally affect the overall particle density. This is in line with the fractional coverage being lower than 0.15 , and therefore the probable absence of loosely bound particles on this surface. Furthermore, particle aggregation was only induced by CEA. In this situation, repulsion due to negative charges of citrates was no longer effective and aggregates formed ${ }^{53}$. On the whole, post-treatment on AuPEG-AuNP by DAPEG allowed maintaining a high density of NP as well as a good dispersion. The nanostructured Si-PEG-AuNP and Au-PEG-AuNP substrates post-functionalized with DAPEG were further selected to build up a piezoelectric immunosensor for the antiinflammatory drug diclofenac.

Anti-diclofenac antibody recognition, specificity and reversibility: Diclofenac was covalently bound through its carboxylic acid function to the PEG amino groups of the nanostructured gold and silicon sensors and recognition of anti-diclofenac antibody was investigated by QCM-D as depicted in Figure 4. QCM was also used to assess the specificity of recognition towards non specific anti-mouse IgG (Supplementary section, Figure S6) as well as the reversibility of the 
hapten / antibody interaction (Supplementary section, Figure S7). In addition, to determine whether nanostructuration benefits to target recognition, the same experiment was run on flat silicon and gold substrates modified by GOPTS-PEG and MUA-PEG, respectively (Figure 4).

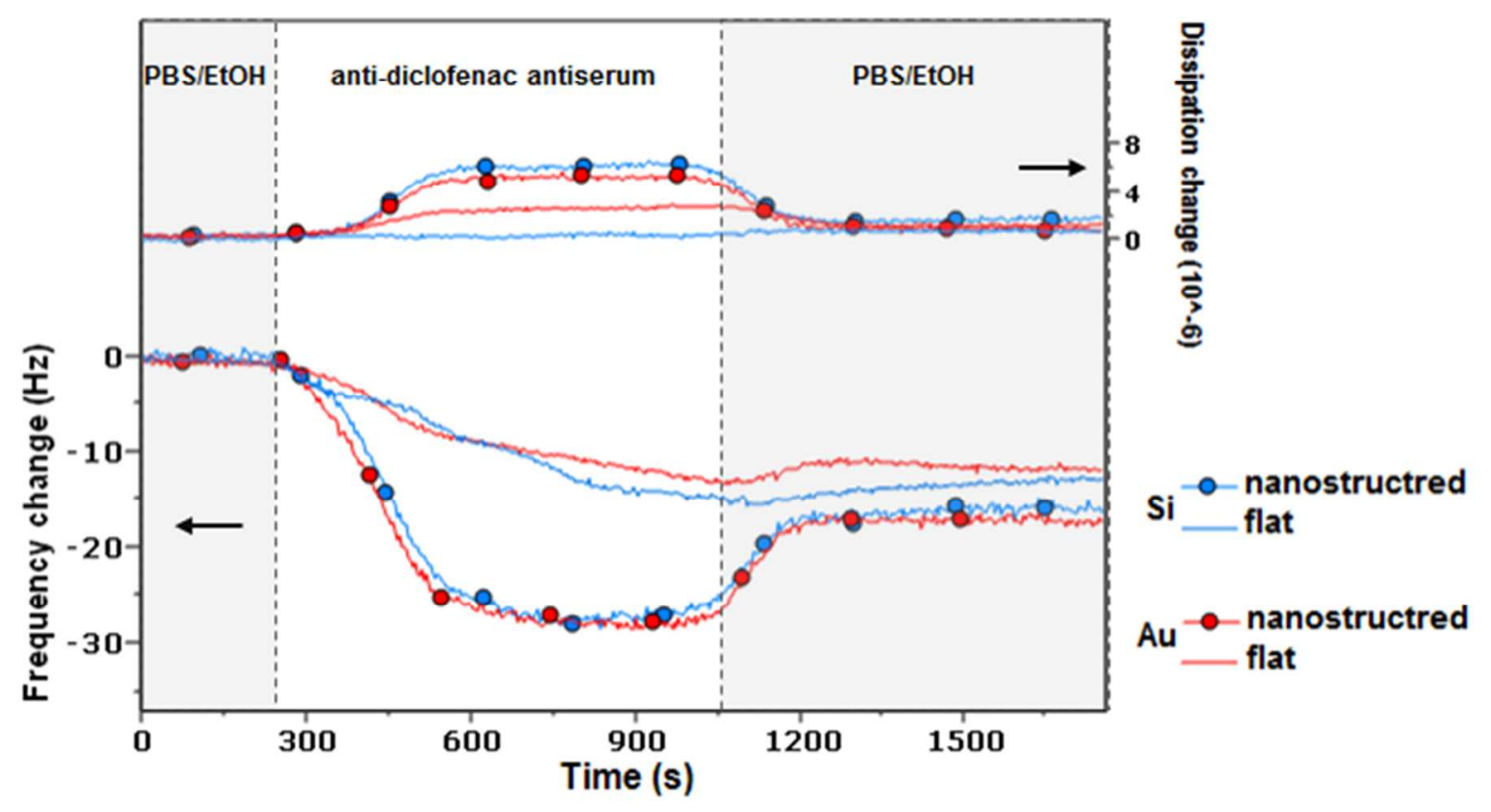

Figure 4 Frequency and dissipation shifts monitored during capture of anti-diclofenac antibody and washing with carrier buffer with the 4 different immunosensors

Injection of non-specific antibody had no influence on the resonance frequency (Figure S6). Conversely, injection of anti-diclofenac antibody resulted in an immediate decrease of the resonance frequency of the 4 sensors confirming the recognition of the specific anti-diclofenac antibody by the capture layer comprising diclofenac (Figure 4). The magnitude of the shift $(\Delta \mathrm{F})$ as well as the rate of frequency change $\mathrm{d} \Delta \mathrm{F} / \mathrm{dt}$ were highly dependent on the nature of the sensing layer. On all systems, applying a regeneration buffer $(\mathrm{pH}=2)$ led to the release of the captured antibodies and return to the initial frequency (Figure S7).

We also analyzed the AuNPs layers by SEM to establish the possible effect of 
immunorecognition and regeneration on their dispersion and coverage. The SEM images in

Figure 5 show that the gold nanoparticles layer was remarkably stable after the biorecognition step and even after the drastic regeneration conditions at acidic $\mathrm{pH}$. No change was recorded upon anti-diclofenac binding to the surface. Upon regeneration, the drastic conditions applied led to a decrease in nanoparticle dispersion, yet, only small aggregates were formed and less than $15 \%$ of AuNP formed aggregates larger than 4 units. The regeneration process needs to be optimized to further limit its impact on AuNP dispersion.
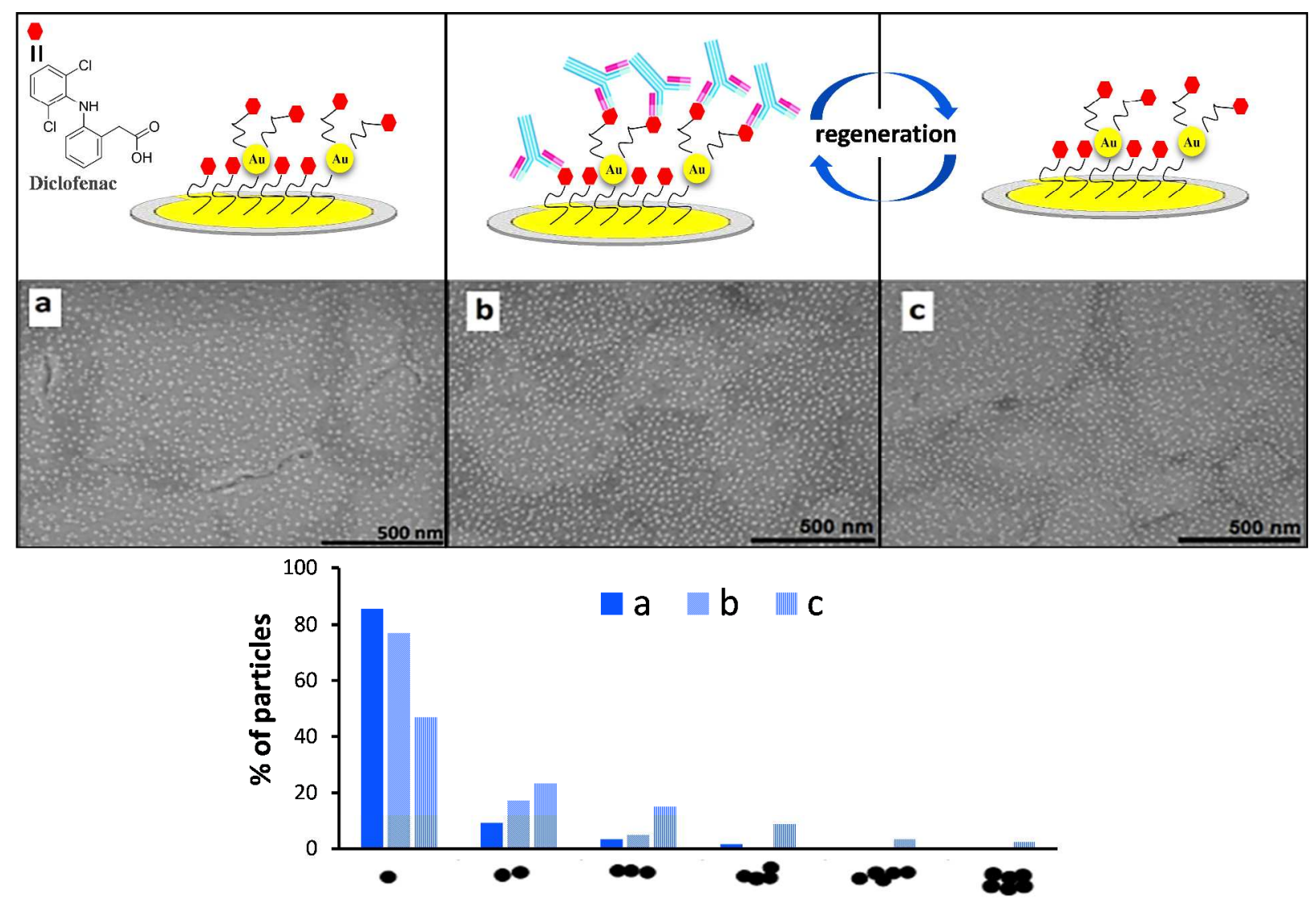

Figure 5 Reversible immunorecognition scheme and SEM images and data of Au-PEG gold nanoparticles platforms post-functionalized with $\mathrm{PEG}_{68}$ at the successive stages of the immunoreaction

Mass uptakes $\Delta \mathrm{m}$ were calculated from the frequency shifts by applying the Sauerbrey equation 
since dissipation shifts were lower that $1 \times 10^{-6}$ during antibody capture (Table 3 ). The QCM responses in terms of $\Delta \mathrm{F}$ and $\mathrm{d} \Delta \mathrm{F} / \mathrm{dt}$ were significantly larger for the 2 nanostructured sensor chips in agreement with the increase of specific surface brought by the layer of AuNPs. Clearly, the two flat sensors had lower antibody binding capacity than the nanostructured ones. Before washing with buffer, the increase was over $120 \%$ for both substrates, i.e. more than 6 times higher than on flat surfaces. After washing, the input of nanostruturation was still important, 32 and $48 \%$ for silicon and gold, respectively. The origin of this enhancement may be geometrical as the nanostructured sensors display a higher specific area than the flat ones. Knowing the surface coverage in AuNPs and their mean size, the increase of accessible surface area can be readily estimated. We have done these calculations depending on the exposed height of AuNPs (i.e. how the AuNPs are actually embedded in the organic film). The increase in area ranged from 17 to $33 \%$ for the Si sensor (with 1073 particles $/ \mu \mathrm{m}^{2}$ ) and from 13 to $26 \%$ for the Au sensor (with 833 particles $/ \mu \mathrm{m}^{2}$ ). Alternatively, the surface topology of nanostructured sensors may also favor the accessibility of the immobilized diclofenac ligand towards its antibody further enhancing the geometric benefit of nanostructuration.

Table 3 QCM data for flat and AuNPs nanostructured surfaces

\begin{tabular}{lcc|cc} 
& \multicolumn{2}{c|}{ Gold } & \multicolumn{2}{c}{ Silicon } \\
\cline { 2 - 5 } & Flat & Nanostructured & Flat & Nanostructured \\
\hline$-\Delta F_{5}(\mathrm{~Hz})$ & 11.8 & $17.5 \pm 1$ & 12.5 & $16.5 \pm 0.5$ \\
$\Delta \mathrm{m} / \mathrm{A}\left(\mathrm{ng} / \mathrm{cm}^{2}\right)$ & 208 & $310 \pm 18$ & 222 & $293 \pm 9$ \\
$\Gamma(\mathrm{exp})\left(\mathrm{pmol} . \mathrm{cm}^{-2}\right)^{*}$ & 1.4 & 2 & 1.5 & 1.95 \\
Increase $(\%)$ before washing & --- & 128 & --- & 124 \\
Increase after washing $(\%)$ & --- & 48 & --- & 32 \\
$-\mathrm{d} \Delta \mathrm{F} / \mathrm{dt}\left(\mathrm{Hz}_{\mathrm{s}} \mathrm{s}^{-1}\right)$ & 0.016 & 0.077 & 0.019 & 0.088
\end{tabular}

Furthermore, the nanostructured surfaces on a silicon or gold substrates gave very similar responses in QCM. The amount of anti-diclofenac antibodies recognizing the target differs little 
despite a difference in the total density of AuNPs greater than $20 \%$ for silicon substrates. This result corroborates the assumption that increasing the surface area is the one and only reason of the improved performances of nanostructured immunosensors and abounds and towards a greater contribution granted by the new surface topology and the increased accessibility of the target. This result thus also proves that, at this stage, the influence of the substrate is limited which paves the way for a generalization of this surface chemistry to other substrates by expecting the same performances.

To conclude this part, AuNPs layers led to a significant enhancement in the biosensor sensitivity. This enhancement presages a higher sensitivity in the competitive detection of diclofenac on these systems. More importantly, despite the biorecognition and the drastic regeneration conditions, gold nanoparticle layers were stable and reliable, which paves the way for their use as nanostructured platforms for various applications.

\section{CONCLUSION}

Different surface chemistries were devised to generate layers of gold nanoparticles (AuNPs) on gold and silicon planar substrates. Adhesion layers either included alkyl chains or poly(ethylene glycol) (PEG) and were terminated by amines or mixed amine and thiol groups. The assembly of gold nanoparticles on the functionalized substrates was studied in depth by Scanning Electron Microscopy. The highest coverage and dispersion were reached when gold nanoparticles were deposited on adhesion layers offering multiple anchoring points and cooperative interactions. These systems showed a resistance to leaching upon sonication which made them suitable for further functionalization. Adhesion layers including PEG motifs provided an excellent coverage and the best dispersion on both Si and Au substrates (78\%). These layers were post- 
functionalized using either PEG-diamine or cysteamine to exchange the surrounding citrate ligands by reactive functions that will be used to further bind the bioreceptors. This step slightly decreased the coverage in gold nanoparticles, particularly on silicon substrates, but the use of PEG-diamine better preserved the dispersion of gold nanoparticles. The resulting layers were successfully used to build up a piezoelectric immunosensor for the anti-inflammatory drug diclofenac. They were sensitive and specific to diclofenac antibody. Compared to planar surfaces, they showed a significant enhancement in the biosensor sensitivity, up to 6 times the signal recorded for planar substrates. More importantly, despite the biorecognition and the drastic regeneration conditions, QCM sensors covered with gold nanoparticle layers were stable and reusable with a preserved coverage and dispersion, which paves the way for their use as nanostructured platforms for various applications.

\section{SUPPORTING INFORMATION}

Gold nanoparticles characterizations. IR characterization of functionalized surfaces. Gold nanoparticles deposition. QCM data.

\section{ACKNOWLEDGEMENTS}

We would like to thank the DIM Analytics and Region Ile-de-France for M. Ben Haddada PhD scholarship. This work was also co-financially supported by ANR (Agence Nationale de la Recherche) and DFG (Deutsche Forschungsgemeinschaft), ANR-DFG program, project NArBioS, Grant no: ANR-11-INTB-1013. 


\section{REFERENCES}

1. Saha, K.; Agasti, S. S.; Kim, C.; Li, X. N.; Rotello, V. M., Gold Nanoparticles in Chemical and Biological Sensing. Chem. Rev. 2012, 112, 2739-2779.

2. Nath, N.; Chilkoti, A., Label-Free Biosensing by Surface Plasmon Resonance of Nanoparticles on Glass: Optimization of Nanoparticle Size. Anal. Chem. 2004, 76, 5370-5378.

3. Sepulveda, B.; Angelome, P. C.; Lechuga, L. M.; Liz-Marzan, L. M., LSPR-Based Nanobiosensors. Nano Today 2009, 4, 244-251.

4. Stewart, M. E.; Anderton, C. R.; Thompson, L. B.; Maria, J.; Gray, S. K.; Rogers, J. A.; Nuzzo, R. G., Nanostructured Plasmonic Sensors. Chem. Rev. 2008, 108, 494-521.

5. Fan, M. K.; Andrade, G. F. S.; Brolo, A. G., A Review on the Fabrication of Substrates for Surface Enhanced Raman Spectroscopy and Their Applications in Analytical Chemistry. Anal. Chim. Acta 2011, 693, 7-25.

6. Felicia, T.; Monica, B.; Lucian, B.; Simion, A., Controlling Gold Nanoparticle Assemblies for Efficient Surface-Enhanced Raman Scattering and Localized Surface Plasmon Resonance Sensors. Nanotechnology 2007, 18, 255702.

7. $\quad$ Fonseca, R. A. S.; Ramos-Jesus, J.; Kubota, L. T.; Dutra, R. F., A Nanostructured Piezoelectric Immunosensor for Detection of Human Cardiac Troponin T. Sensors 2011, 11, 10785-10797.

8. $\quad$ Kong, L.-J.; Pan, M.-F.; Fang, G.-Z.; He, X.-1.; Yang, Y.-k.; Dai, J.; Wang, S., Molecularly Imprinted Quartz Crystal Microbalance Sensor Based on Poly(O-Aminothiophenol) Membrane and Au Nanoparticles for Ractopamine Determination. Biosens. Bioelectron. 2014, 51, 286-292.

9. Cai, H.; Xu, C.; He, P.; Fang, Y., Colloid Au-Enhanced DNA Immobilization for the Electrochemical Detection of Sequence-Specific DNA. J. Electroanal. Chem. 2001, 510, 78-85.

10. Liu, T.; Tang, J. a.; Jiang, L., The Enhancement Effect of Gold Nanoparticles as a Surface Modifier on DNA Sensor Sensitivity. Biochem. Biophys. Res. Commun. 2004, 313, 3-7.

11. Liu, S.-f.; Li, J.-R.; Jiang, L., Surface Modification of Platinum Quartz Crystal Microbalance by Controlled Electroless Deposition of Gold Nanoparticles and Its Enhancing Effect on the Hs-DNA Immobilization. Colloids Surf. Physicochem. Eng. Aspects 2005, 257258, 57-62.

12. Chu, P.-T.; Lin, C.-S.; Chen, W.-J.; Chen, C.-F.; Wen, H.-W., Detection of Gliadin in Foods Using a Quartz Crystal Microbalance Biosensor That Incorporates Gold Nanoparticles. $J$. Agric. Food. Chem. 2012, 60, 6483-6492.

13. Vidal, J. C.; Duato, P.; Bonel, L.; Castillo, J. R., Use of Polyclonal Antibodies to Ochratoxin a with a Quartz-Crystal Microbalance for Developing Real-Time Mycotoxin Piezoelectric Immunosensors. Anal. Bioanal. Chem. 2009, 394, 575-582.

14. Wang, H.; Liu, Y. L.; Yang, Y. H.; Deng, T.; Shen, G. L.; Yu, R. Q., A Protein a-Based Orientation-Controlled Immobilization Strategy for Antibodies Using Nanometer-Sized Gold Particles and Plasma-Polymerized Film. Anal. Biochem. 2004, 324, $219-226$.

15. Wang, H.; Wu, J.; Li, J.; Ding, Y.; Shen, G.; Yu, R., Nanogold Particle-Enhanced Oriented Adsorption of Antibody Fragments for Immunosensing Platforms. Biosens. Bioelectron. 2005, 20, 2210-2217.

16. Makaraviciute, A.; Ruzgas, T.; Ramanavicius, A.; Ramanaviciene, A., Antibody Fragment Immobilization on Planar Gold and Gold Nanoparticle Modified Quartz Crystal Microbalance with Dissipation Sensor Surfaces for Immunosensor Applications. Anal. Meth. 2014, 6, $2134-$ 2140 . 
17. Turkevich, J.; Stevenson, P. C.; Hillier, J., A Study of the Nucleation and Growth Processes in the Synthesis of Colloidal Gold. Farad. Disc. 1951, 55-\&.

18. Morel, A.-L.; Boujday, S.; Méthivier, C.; Krafft, J.-M.; Pradier, C.-M., Biosensors Elaborated on Gold Nanoparticles, a PM-IRRAS Characterisation of the IgG Binding Efficiency. Talanta 2011, 85, 35-42.

19. Morel, A.-L.; Volmant, R.-M.; Méthivier, C.; Krafft, J.-M.; Boujday, S.; Pradier, C.-M., Optimized Immobilization of Gold Nanoparticles on Planar Surfaces through Alkyldithiols and Their Use to Build 3D Biosensors. Colloids Surf., B 2010, 81, 304-312.

20. Ben Haddada, M., Optimizing the Immobilization of Gold Nanoparticles on Functionalized Silicon Surfaces: Amine- Vs Thiol-Terminated Silane. Gold Bulletin 2013, 1-7. 21. Aissaoui, N.; Bergaoui, L.; Landoulsi, J.; Lambert, J.-F.; Boujday, S., Silane Layers on Silicon Surfaces: Mechanism of Interaction, Stability, and Influence on Protein Adsorption. Langmuir 2012, 28, 656-665.

22. Christau, S.; Moller, T.; Yenice, Z.; Genzer, J.; von Klitzing, R., Brush/Gold Nanoparticle Hybrids: Effect of Grafting Density on the Particle Uptake and Distribution within Weak Polyelectrolyte Brushes. Langmuir 2014, 30, 13033-13041.

23. Christau, S.; Thurandt, S.; Yenice, Z.; von Klitzing, R., Stimuli-Responsive Polyelectrolyte Brushes as a Matrix for the Attachment of Gold Nanoparticles: The Effect of Brush Thickness on Particle Distribution. Polymers 2014, 6, 1877-1896.

24. Mehne, J.; Markovic, G.; Pröll, F.; Schweizer, N.; Zorn, S.; Schreiber, F.; Gauglitz, G., Characterisation of Morphology of Self-Assembled Peg Monolayers: A Comparison of Mixed and Pure Coatings Optimised for Biosensor Applications. Anal. Bioanal. Chem. 2008, 391, 17831791.

25. Huebner, M.; Ben Haddada, M.; Methivier, C.; Niessner, R.; Knopp, D.; Boujday, S., Layer-by-Layer Generation of Peg-Based Regenerable Immunosensing Surfaces for Small-Sized Analytes. Biosens. Bioelectron. 2015, 67, 334-341.

26. Diamanti, S.; Arifuzzaman, S.; Genzer, J.; Vaia, R. A., Tuning Gold Nanoparticle-Poly(2Hydroxyethyl Methacrylate) Brush Interactions: From Reversible Swelling to Capture and Release. ACS Nano 2009, 3, 807-818.

27. Meyerbröker, N.; Kriesche, T.; Zharnikov, M., Novel Ultrathin Poly(Ethylene Glycol) Films as Flexible Platform for Biological Applications and Plasmonics. ACS Appl. Mater. Interfaces 2013, 5, 2641-2649.

28. Daniel, M. C.; Astruc, D., Gold Nanoparticles: Assembly, Supramolecular Chemistry, Quantum-Size-Related Properties, and Applications toward Biology, Catalysis, and Nanotechnology. Chem. Rev. 2004, 104, 293-346.

29. Brust, M.; Fink, J.; Bethell, D.; Schiffrin, D. J.; Kiely, C., Synthesis and Reactions of Functionalized Gold Nanoparticles. Chem. Commun. 1995, 1655-1656.

30. Pena-Pereira, F.; Duarte, R. M. B. O.; Duarte, A. C., Immobilization Strategies and Analytical Applications for Metallic and Metal-Oxide Nanomaterials on Surfaces. TrAC, Trends Anal. Chem. 2012, 40, 90-105.

31. Lundgren, A.; Hulander, M.; Brorsson, J.; Hermansson, M.; Elwing, H.; Andersson, O.; Liedberg, B.; Berglin, M., Gold-Nanoparticle-Assisted Self-Assembly of Chemical Gradients with Tunable Sub-50 Nm Molecular Domains. Part. Part. Syst. Charact. 2014, 31, 209-218. 32. Deng, A. P.; Himmelsbach, M.; Zhu, Q. Z.; Frey, S.; Sengl, M.; Buchberger, W.; Niessner, R.; Knopp, D., Residue Analysis of the Pharmaceutical Diclofenac in Different Water Types Using Elisa and Gc-Ms. Environ. Sci. Technol. 2003, 37, 3422-3429. 
33. Huebner, M.; Weber, E.; Niessner, R.; Boujday, S.; Knopp, D., Rapid Analysis of Diclofenac in Freshwater and Wastewater by a Monoclonal Antibody-Based Highly Sensitive Elisa. Anal. Bioanal. Chem. 2015, 407, 8873-8882.

34. Cras, J. J.; Rowe-Taitt, C. A.; Nivens, D. A.; Ligler, F. S., Comparison of Chemical Cleaning Methods of Glass in Preparation for Silanization. Biosens. Bioelectron. 1999, 14, 683688.

35. Aissaoui, N.; Bergaoui, L.; Landoulsi, J.; Lambert, J. F.; Boujday, S., Silane Layers on Silicon Surfaces: Mechanism of Interaction, Stability, and Influence on Protein Adsorption. Langmuir 2012, 28, 656-65.

36. Slot, J. W.; Geuze, H. J., A Method to Prepare Isodisperse Colloidal Gold Sols in the Size Range 3-17 Nm. Ultramicroscopy 1984, 15, 383.

37. Boujday, S.; Gu, C.; Girardot, M.; Salmain, M.; Pradier, C.-M., Surface Ir Applied to Rapid and Direct Immunosensing of Environmental Pollutants. Talanta 2009, 78, 165-170.

38. Sauerbrey, G., The Use of Quartz Oscillators for Weighing Thin Layers and for Microweighing. Z. Phys. 1959, 155, 206-22.

39. Seitz, O.; Chehimi, M. M.; Cabet-Deliry, E.; Truong, S.; Felidj, N.; Perruchot, C.; Greaves, S. J.; Watts, J. F., Preparation and Characterisation of Gold Nanoparticle Assemblies on Silanised Glass Plates. Colloids Surf., A 2003, 218, 225-239.

40. Link, S.; El-Sayed, M. A., Size and Temperature Dependence of the Plasmon Absorption of Colloidal Gold Nanoparticles. J. Phys. Chem. B 1999, 103, 4212-4217.

41. Grabar, K. C.; Freeman, R. G.; Hommer, M. B.; Natan, M. J., Preparation and Characterization of Au Colloid Monolayers. Anal. Chem. 1995, 67, 735-743.

42. Onses, M. S.; Nealey, P. F., Tunable Assembly of Gold Nanoparticles on Nanopatterned Poly(Ethylene Glycol) Brushes. Small 2013, 9, 4168-4174.

43. Kanan, S. M.; Tze, W. T. Y.; Tripp, C. P., Method to Double the Surface Concentration and Control the Orientation of Adsorbed (3-Aminopropyl)Dimethylethoxysilane on Silica Powders and Glass Slides. Langmuir 2002, 18, 6623-6627.

44. Vericat, C.; Vela, M. E.; Salvarezza, R. C., Self-Assembled Monolayers of Alkanethiols on Au(111): Surface Structures, Defects and Dynamics. PCCP 2005, 7, 3258-3268.

45. Vallee, A.; Humblot, V.; Al Housseiny, R.; Boujday, S.; Pradier, C.-M., Bsa Adsorption on Aliphatic and Aromatic Acid Sams: Investigating the Effect of Residual Surface Charge and Sublayer Nature. Colloids Surf., B 2013, 109, 136-142.

46. Harder, P.; Grunze, M.; Dahint, R.; Whitesides, G. M.; Laibinis, P. E., Molecular Conformation in Oligo(Ethylene Glycol)-Terminated Self-Assembled Monolayers on Gold and Silver Surfaces Determines Their Ability to Resist Protein Adsorption. J. Phys. Chem. B 1998, $102,426-436$.

47. Nath, N.; Chilkoti, A., Label-Free Biosensing by Surface Plasmon Resonance of Nanoparticles on Glass: Optimization of Nanoparticle Size. Anal. Chem. 2004, 76, 5370-5378. 48. Bedford, E.; Humblot, V.; Methivier, C.; Pradier, C.-M.; Gu, F.; Tielens, F.; Boujday, S., An Experimental and Theoretical Approach to Investigate the Effect of Chain Length on Aminothiol Adsorption and Assembly on Gold. Chem. Eur. J. 2015, 21, 14555-14561.

49. Riauba, L.; Niaura, G.; Eicher-Lorka, O.; Butkus, E., A Study of Cysteamine Ionization in Solution by Raman Spectroscopy and Theoretical Modeling. J. Phys. Chem. A 2006, 110, 13394-13404.

50. Bhat, R. R.; Genzer, J., Tuning the Number Density of Nanoparticles by Multivariant Tailoring of Attachment Points on Flat Substrates. Nanotechnology 2007, 18. 
51. Iler, R. K., The Chemistry of Silica; Wiley-Interscience: New York, 1979.

52. Enders, D.; Nagao, T.; Pucci, A.; Nakayama, T., Reversible Adsorption of Au Nanoparticles on Sio2/Si: An in Situ Atr-Ir Study. Surf. Sci. 2006, 600, L71-L75.

53. Sato, T.; Ahmed, H.; Brown, D.; Johnson, B. F. G., Single Electron Transistor Using a Molecularly Linked Gold Colloidal Particle Chain. J. Appl. Phys. 1997, 82, 696-701.

54. Sato, T.; Brown, D.; Johnson, B. F. G., Nucleation and Growth of Nano-Gold Colloidal Lattices. Chem. Commun. 1997, 1007-1008.

55. Enders, D.; Nagao, T.; Nakayama, T.; Aono, M., In Situ Surface-Enhanced Infrared Absorption Spectroscopy for the Analysis of the Adsorption and Desorption Process of $\mathrm{Au}$ Nanoparticles on the SiO2/Si Surface. Langmuir 2007, 23, 6119-6125. 
1

2

3

4

5

6

7

8

9

10

11

12

13

14

15

16

17

18

19

20

21

22

23

24

25

26

27

28

29

30

31

32

33

34

35

36

37

38

39

40

41

42

43

44

45

46

47

48

49

50

51

52

53

54

55

56

57

58

59

60

TOC Graphic

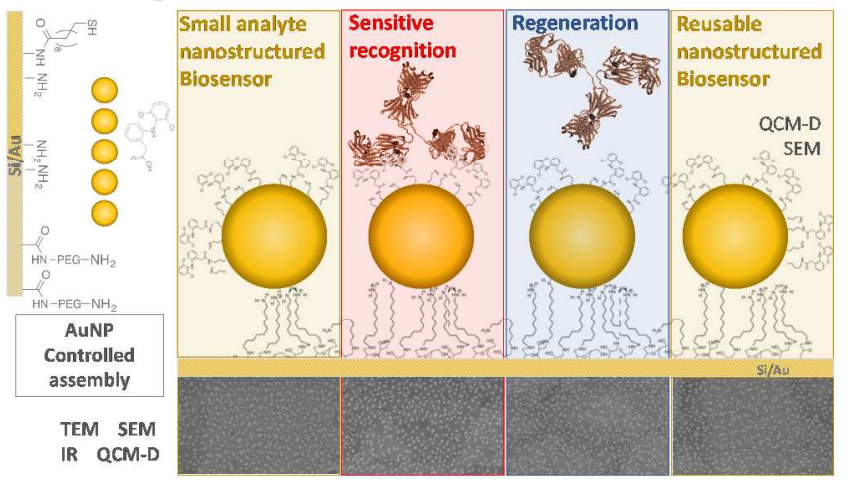

ACS Paragon Plus Environment 


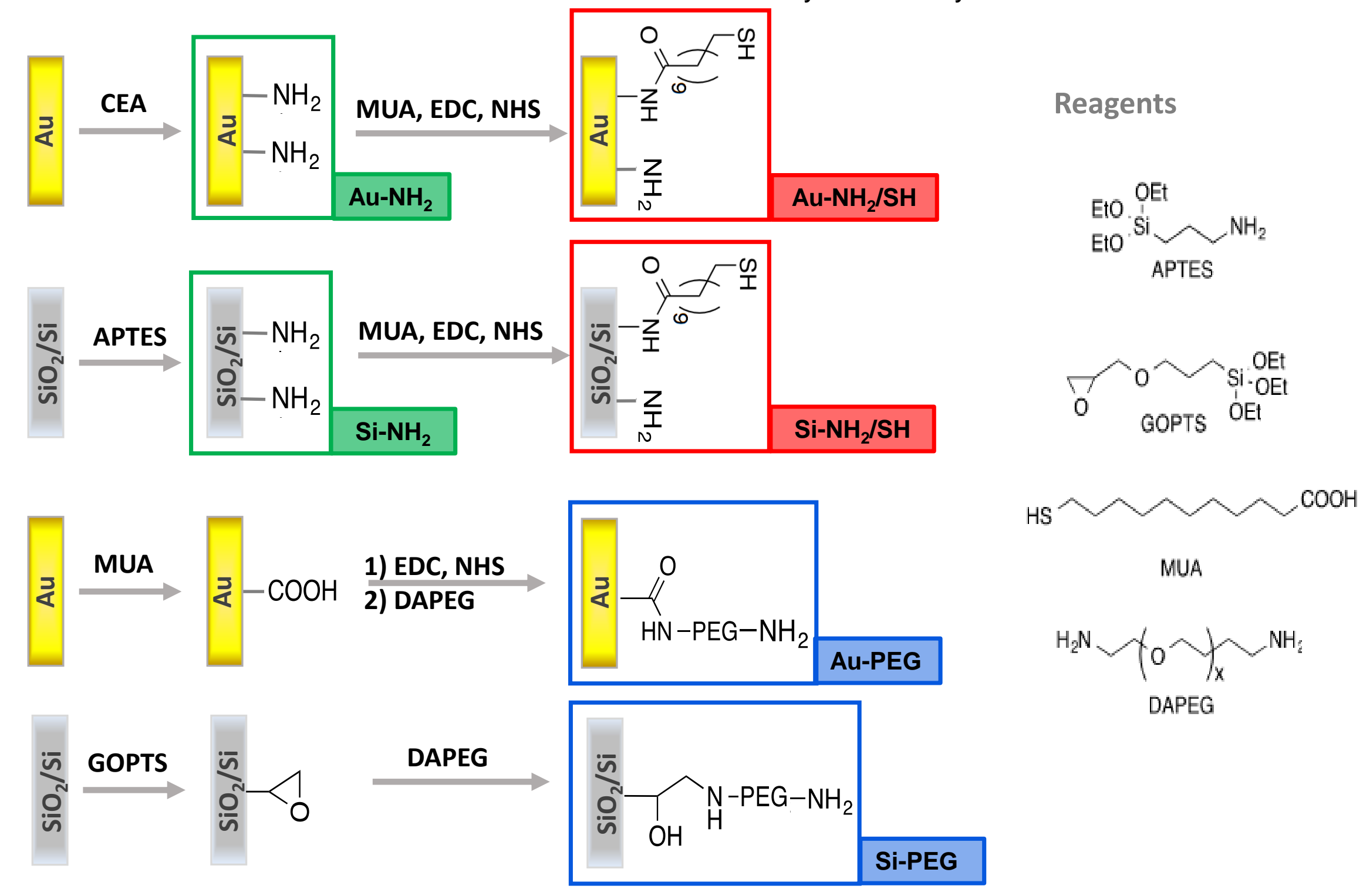




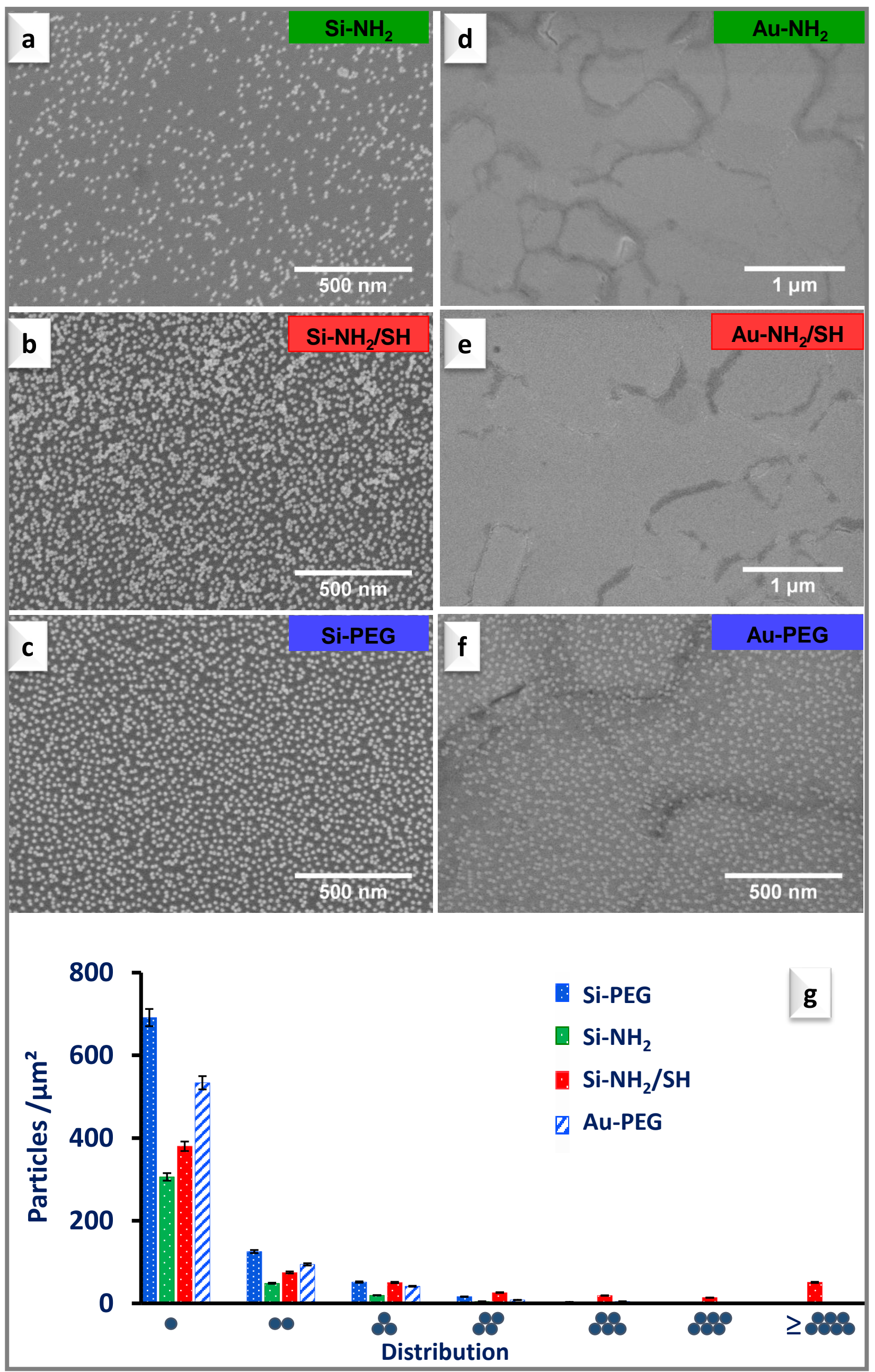




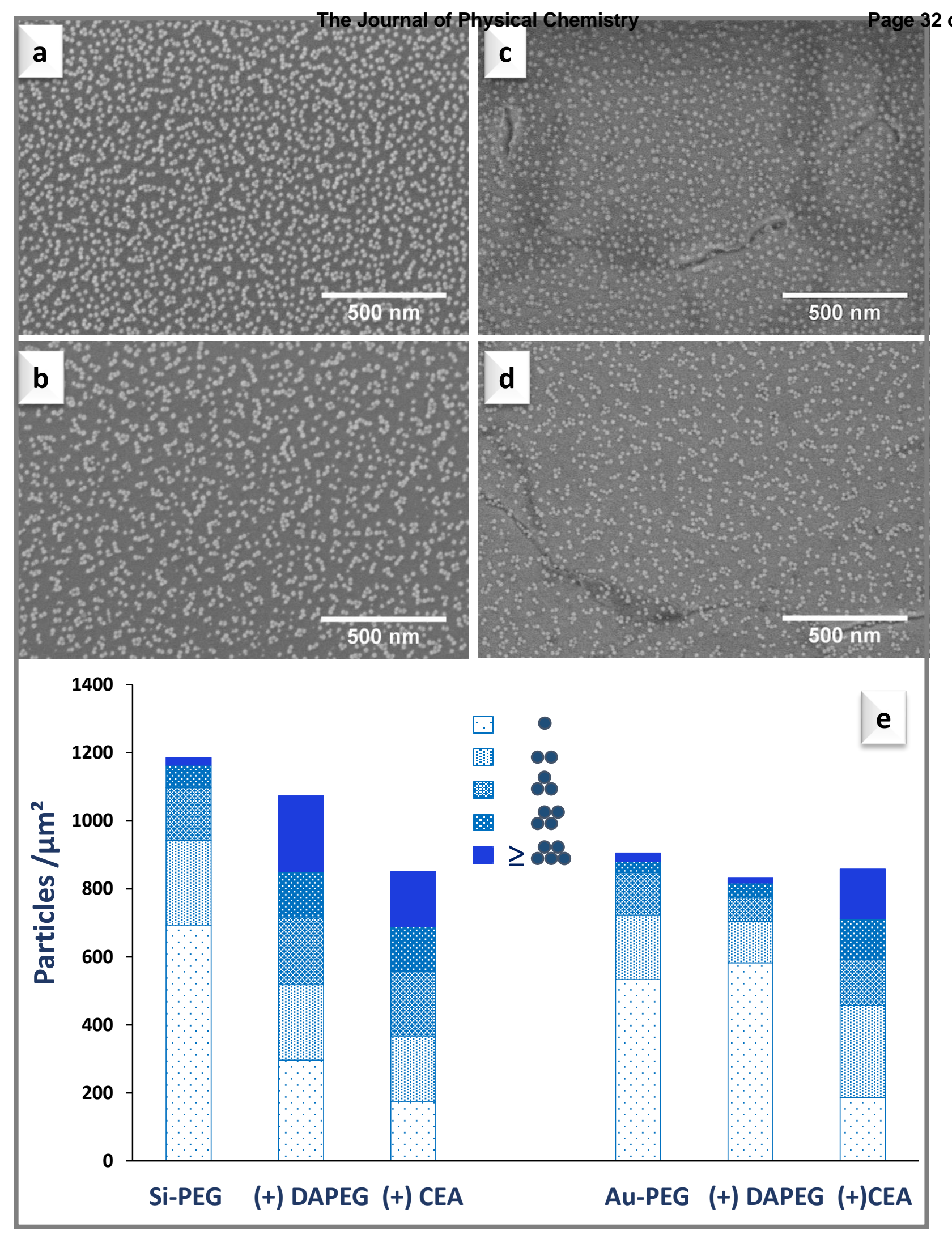




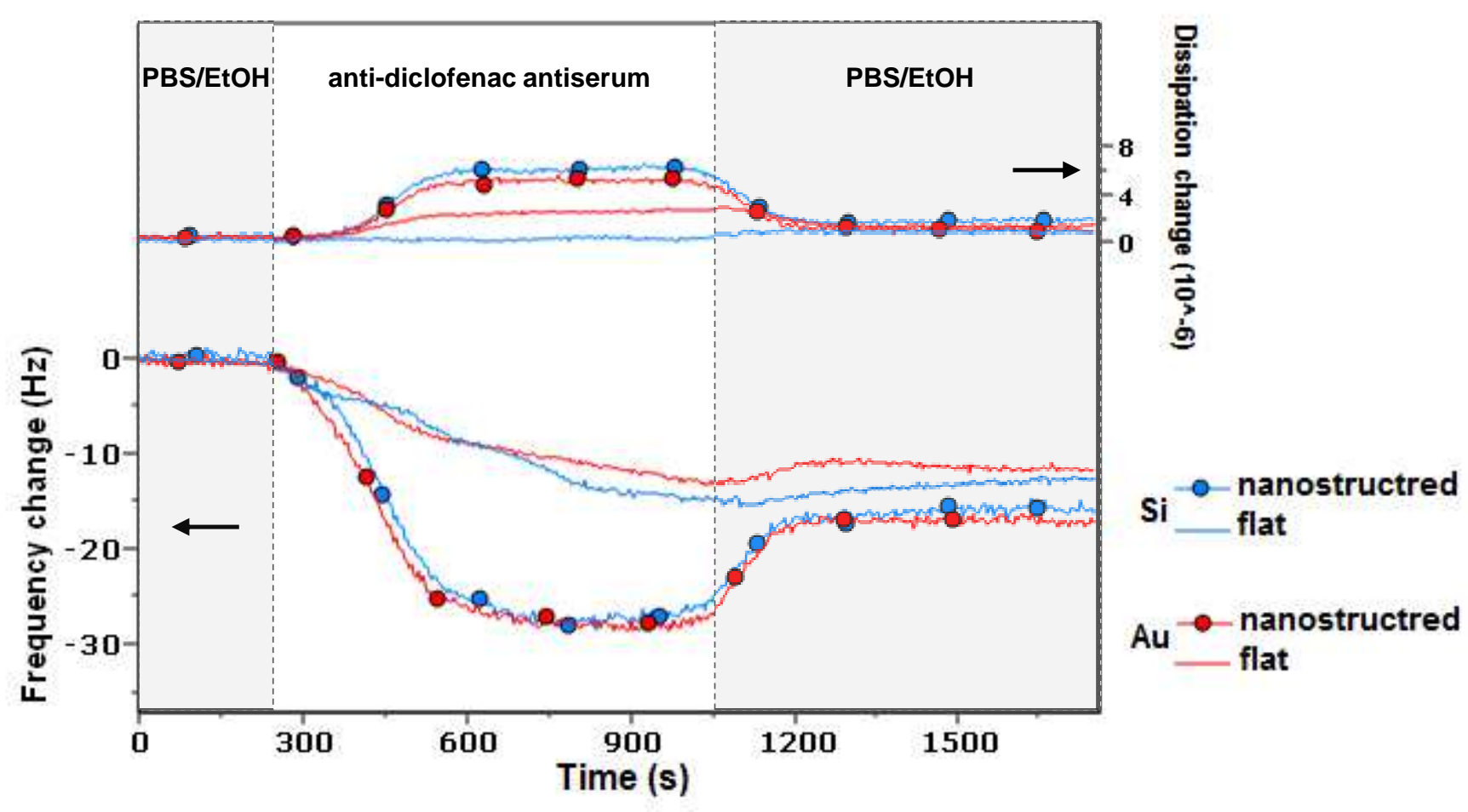




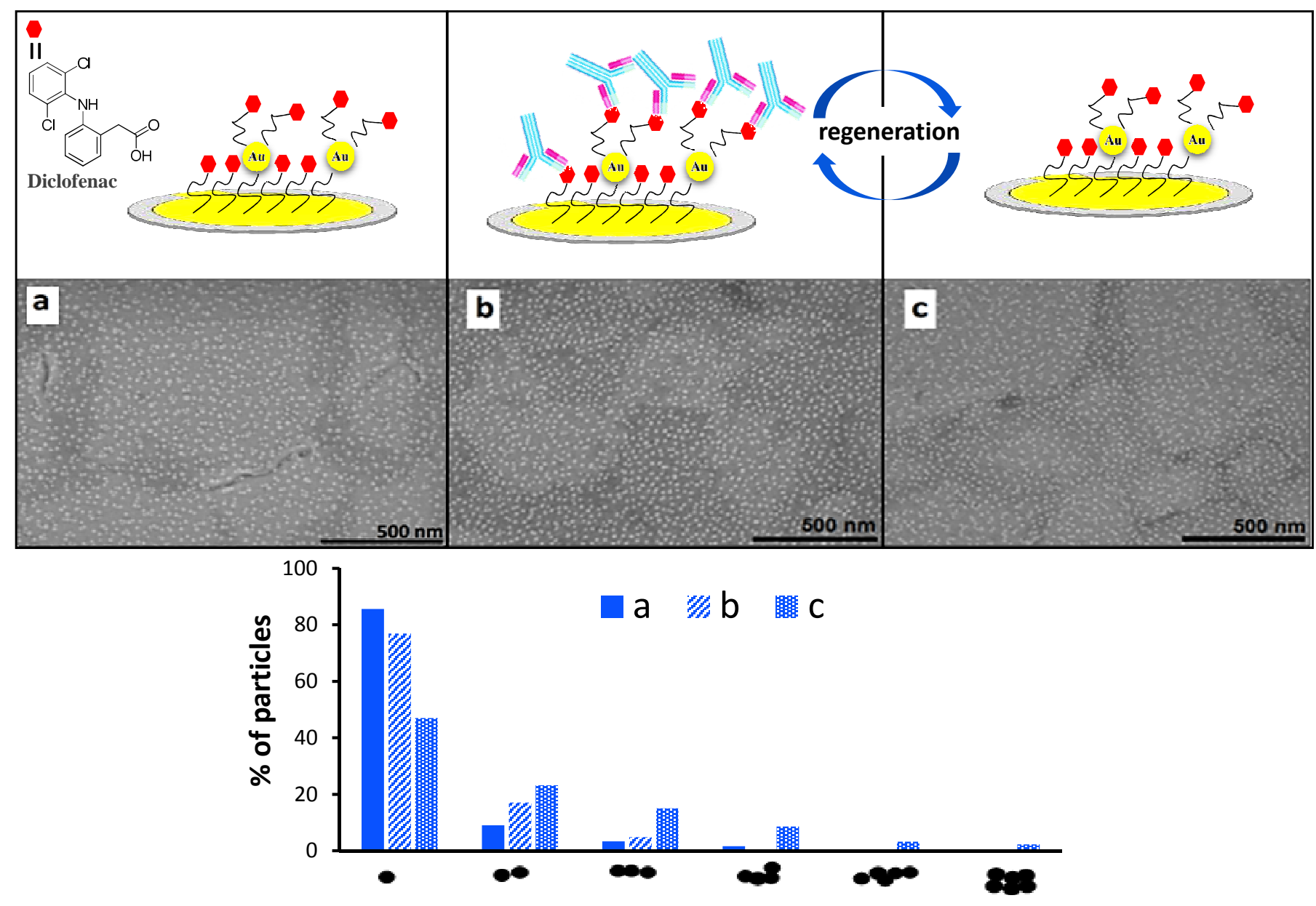

ACS Paragon Plus Environment 


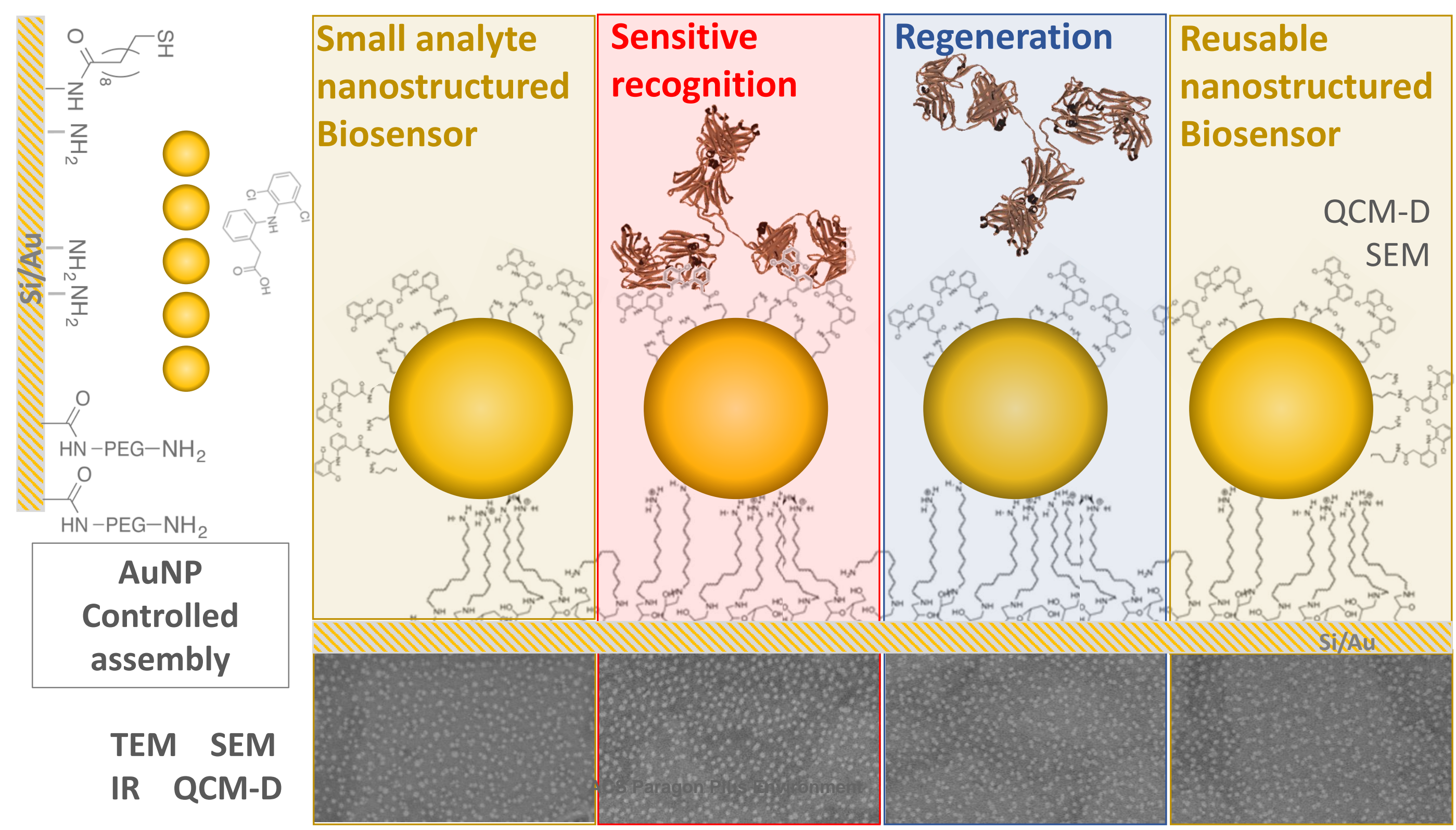

